\title{
Apolipoprotein E Gene Polymorphism and Coronary Artery Disease Risk Among Patients in Northwest China
}

\author{
Wenbing $M a \mathbb{D}^{1,2}$ \\ Xiaodong Ren' \\ Liting Zhang' \\ Haiyan Dong' \\ Xiaoyun $\mathrm{Lu}^{2}$ \\ Weiyi Feng'
}

'Department of Pharmacology, The First Affiliated Hospital of Xi'an Jiaotong University, Xi'an, Shaanxi, 71006I, People's Republic of China; ${ }^{2}$ Department of Biological Science and Bioengineering, Key Laboratory of Biomedical Information Engineering of the Ministry of Education, School of Life Science and Technology, Xi'an Jiaotong University, Xi'an, Shaanxi, 710049, People's Republic of China
Correspondence: Xiaoyun Lu

$\mathrm{Tel} / \mathrm{Fax}+86-29-82668463$

Email luxy05@xitu.edu.cn

Weiyi Feng

Tel/Fax +86-29-85323242

Email fengweiyi@mail.xjtu.edu.cn
Purpose: The association between apolipoprotein E (ApoE) gene polymorphisms and the risk of coronary artery disease (CAD) among different populations has been assessed in numerous previous studies, but the results remain inconclusive. The present study aimed to determine the role of ApoE genotypes in CAD risk and the interrelationships between lipid profiles and ApoE alleles and genotypes among the population of northwest China.

Patients and Methods: This study was performed on 308 patients with CAD and 308 control participants. ApoE gene polymorphism was analysed using the polymerase chain reaction and hybridization.

Results: The findings indicated that the frequencies of $\varepsilon 3 / \varepsilon 4$ genotype and $\varepsilon 4$ allele frequency were significantly higher in patients with CAD than in the control participants. $\varepsilon 2$ carriers had significantly lower total cholesterol (TC), low-density lipoprotein cholesterol (LDL-C) and triglycerides (TG) levels than did $\varepsilon 3$ or $\varepsilon 4$ carriers among the control participants. However, our study found no significant differences in plasma lipoprotein levels between $\varepsilon 2, \varepsilon 3$ and $\varepsilon 4$ carriers in patients with CAD. Moreover, $\varepsilon 4$ carriers had significantly higher ApoB, ApoB/ApoA-I levels and significantly lower ApoE levels in both patients with $\mathrm{CAD}$ and control participants. $\varepsilon 4$ allele presence was associated with a nearly two-fold higher CAD risk. After adjusting for other established risk factors, $\varepsilon 4$ allele was an independent risk factor for CAD. After stratified by age ( $\leq 60$ years and $>60$ years), $\varepsilon 4$ allele was indicated to increase the CAD risk 3.3-fold in elderly patients with $\mathrm{CAD}$, but not in young patients with CAD. After stratified by sex, $\varepsilon 4$ allele was not a risk factor in females and males patients with CAD.

Conclusion: This study provides evidence that the $\varepsilon 4$ allele, drinking, smoking, hypertension, and TG and ApoE levels are independent risk factor for CAD among patients in northwest China.

Keywords: apolipoprotein E, coronary artery disease, gene polymorphism, Northwest China

\section{Introduction}

CAD is a diseases with one of the highest morbidities and mortalities worldwide. ${ }^{1}$ Despite great advances in the clinical management of CAD, atherosclerosis pathogenesis, which underlies CAD, is not fully understood. CAD is a multifactorial disease that results from interactions between genetic variants and environmental factors such as diet, smoking and exercise. Dyslipidemia is known to be a major risk factor for $\mathrm{CAD}$ development. ${ }^{2}$ Lipoproteins play a major role in the development and pathogenesis of atherosclerotic cardiovascular diseases in humans. Plasma lipoprotein levels are largely genetically determined by apolipoprotein. ${ }^{3}$ 
Mutations apolipoprotein gene encoding may result in impaired lipoprotein clearance. Genetic variation may therefore be the main determinant of variation between individuals regarding the susceptibility to CAD. ${ }^{4}$

ApoE is an important plasma protein involved in metabolism and transport of lipids including TC and TG. The polymorphic human ApoE gene, located on chromosome 19q13.2 and SNPs at positions 112 (rs429358) and 158 (rs7412), causes three different alleles ( $\varepsilon 2, \varepsilon 3$ and $\varepsilon 4)$ to encode the major ApoE isoforms ApoE2, ApoE3 and ApoE4, respectively. Numerous studies have indicated that these ApoE gene polymorphisms affect lipoprotein clearing and lipid profiles, which may be responsible for $\mathrm{CAD}$; however, the findings have been inconsistent. ${ }^{2,5}$

ApoE gene polymorphisms affect lipoprotein clearing and lipid profile, and also the development of CAD. ${ }^{6,7}$ Lipid metabolism is influenced by ApoE genotypes. A previous study found that $\varepsilon 4$ carriers can increase CAD risk by approximately $42 \% .{ }^{8} \varepsilon 4$ carriers tend to have higher TC and low-density lipoprotein cholesterol (LDL-C) levels, and exhibit a worse response to statin treatment than do $\varepsilon 2$ carriers. ${ }^{9}$ The distribution of ApoE allele frequencies varies between racial groups, with Europeans and African-Americans having a high $\varepsilon 4$ frequency and Asians having low frequencies of both $\varepsilon 2$ and $\varepsilon 4 .^{2}$ Furthermore, no published studies have investigated the relationship between ApoE polymorphism and CAD risk in northwest China. The present study therefore aimed to determine the role of ApoE genotypes in CAD risk and the interrelationship between lipid profiles and ApoE alleles and genotypes among the population of northwest China.

\section{Methods}

\section{Participants}

This study recruited 616 individuals, which consisted of 308 CAD patients (males: females $=223: 85$ ) and 308 individuals with non CAD (males: females=208:100) as healthy controls. Patients and controls involved in this study were collected in First Affiliated Hospital of Xi'an Jiao Tong University from May 2018 to May 2019; Ages ranged from 15 to 93 years. All subjects were unrelated Han Chinese and residents living in Northwest China. CAD was defined as stenosis of $\geq 50 \%$ in the left main coronary artery, the left anterior descending artery, the left circumflex coronary artery, the right coronary artery or the main branch of the coronary artery. The control groups included participants without stenosis confirmed by coronary angiography and healthy individuals without cardiovascular diseases identified by physical examination. Hypertension was defined as a blood pressure of $\geq 140 / 90$ $\mathrm{mmHg}$ or currently receiving hypertension treatment. Diabetes mellitus was defined as fasting glucose $\geq 7.0$ $\mathrm{mmol} / \mathrm{L}$, or current treatment with oral hypoglycemic agents or insulin. Exclusion criteria included liver disease, and relevant brain lesions detected on MRI. This study was conducted in accordance with the Declaration of Helsinki, approved by the Ethical Committee of the First Affiliated Hospital of Xi' an Jiao Tong University, and we also got the informed consent of each patients.

\section{DNA Extraction and Genotyping}

Blood samples (approximately $4 \mathrm{~mL}$ ) were collected from study populations and stored in tubes containing ethylene diamine tetraacetic acid (EDTA). Genomic DNA was extracted from peripheral blood using a commercial isolation kit (Sinochips Bioscience Co., Ltd., Zhuhai, Guangdong, China) and stored at $-20^{\circ} \mathrm{C}$. The genotypes of ApoE were detected by a commercially available kit (Sinochips Bioscience Co., Ltd., Zhuhai, Guangdong, China). According to the manufacturer's instructions. PCR was performed:

$50^{\circ} \mathrm{C}$ for two minutes, pre-denaturation at $95^{\circ} \mathrm{C}$ for 15 minutes, followed by 45 cycles at $94^{\circ} \mathrm{C}$ for 30 seconds and $65^{\circ} \mathrm{C}$ for 45 seconds. After PCR amplification, the PCR products were then reversely hybridized with gene chip technology. Finally, a gene chip scanner was used to interpret the data.

\section{Statistical Analyses}

All statistical analysis were performed using SPSS version 16.0. Data are presented as means \pm standard deviation for continuous variables and as numbers and percentages for categorical variables. Differences between each group were performed using Student's $t$-test for continuous variables, and Pearson's $\chi^{2}$ test for categorical variables.

The association between disease and risk factors were screened by univariate logistic regression analysis, and odds ratio (OR), with $95 \%$ confidence intervals (CI) was adjusted by multivariate binary logistic regression. ApoE genotype and allele frequencies were tested for HardyWeinberg equilibrium by chi-square test. All tests were two-sided and a $P$ value $<0.05$ was considered. 
Table I Characteristics of the Study Population

\begin{tabular}{|l|c|c|c|}
\hline Characteristics & $\begin{array}{c}\text { Controls } \\
(\mathbf{n = 3 0 8 )}\end{array}$ & $\begin{array}{c}\text { CAD } \\
(\mathbf{n}=308)\end{array}$ & P value \\
\hline Age(years) & $56.91 \pm 14.03$ & $57.76 \pm 12.38$ & $0.423^{\mathrm{a}}$ \\
Males/Females & $208 / 100$ & $223 / 85$ & $0.187^{\mathrm{b}}$ \\
Smoking & $105(30.09 \%)$ & $145(47.08 \%)$ & $0.00 \mathrm{I}^{\mathrm{b}}$ \\
Drinking & $62(20.13 \%)$ & $106(34.42 \%)$ & $<0.00 \mathrm{I}^{\mathrm{b}}$ \\
Diabetes & $76(24.68 \%)$ & $104(33.77 \%)$ & $0.013^{\mathrm{b}}$ \\
Hypertension & $15 \mathrm{I}(49.03 \%)$ & $206(66.88 \%)$ & $<0.00 \mathrm{I}^{\mathrm{b}}$ \\
TC & $3.79 \pm 0.84$ & $3.77 \pm 1.08$ & $0.773^{\mathrm{a}}$ \\
HDL & $1.01 \pm 0.26$ & $0.95 \pm 0.20$ & $0.003^{\mathrm{a}}$ \\
LDL-C & $2.27 \pm 0.74$ & $2.21 \pm 0.98$ & $0.447^{\mathrm{a}}$ \\
TG & $1.48 \pm 0.88$ & $1.69 \pm 1.21$ & $0.021^{\mathrm{a}}$ \\
Cr & $64.12 \pm 20.97$ & $67.50 \pm 18.20$ & $0.041^{\mathrm{a}}$ \\
ApoA-I & $1.16 \pm 0.20$ & $1.12 \pm 0.19$ & $0.009^{\mathrm{a}}$ \\
ApoB & $0.77 \pm 0.21$ & $0.76 \pm 0.24$ & $0.645^{\mathrm{a}}$ \\
ApoB/ApoA-I & $0.68 \pm 0.21$ & $0.70 \pm 0.24$ & $0.375^{\mathrm{a}}$ \\
ApoE & $38.20 \pm 14.97$ & $35.14 \pm 15.85$ & $0.018^{\mathrm{a}}$ \\
\hline
\end{tabular}

Notes: ${ }^{\text {ap }} P$ values were calculated by Student's $t$-tests. ${ }^{\mathrm{b}} P$ values were calculated from two-sided chi-square test.

Abbreviations: CAD, coronary artery disease; TC, total cholesterol; HDL, highdensity lipoproteins; LDL-C, low-density lipoprotein cholesterol; TG, triglyceride; $\mathrm{Cr}$, creatinine.

\section{Results}

\section{Baseline Clinical Characteristics of the Study Participants}

Table 1 lists the demographic and clinical characteristics of all participants included in this study. There were 308 control participants (208 males and 100 females aged
$56.91 \pm 14.03$ years, mean \pm SD) and 308 patients with CAD (223 males and 85 females, age $57.76 \pm 12.38$ years). The age and sex distributions did not differ significantly between the two groups $(P=0.423$ and 0.187 , respectively). Regarding clinical characteristics, smoking, drinking, diabetes mellitus and hypertension prevalence differed significantly between the patients with CAD and the control participants, as did high-density lipoprotein (HDL), TG, creatinine (Cr), ApoA-I and ApoE levels (all $\mathrm{P}<0.05)$. However, there were no significant differences in the TC, LDL-C, ApoB and ApoB/ApoA-I levels between the two groups (all $P>0.05$ ).

\section{ApoE Polymorphism and CAD Risk}

The distributions of ApoE genotypes and alleles are listed in Table 2. The ApoE genotype frequency distributions in patients with CAD and control participants were concordant with the expectations of the Hardy-Weinberg equilibrium $\left(\chi^{2}=1.35\right.$ and $P=0.85$, and $\chi^{2}=6.41$ and $P=0.17$, respectively). In patients with $\mathrm{CAD}$ and controls participants, $\varepsilon 3 / \varepsilon 3$ accounted for $66.6 \%$ and $74.7 \%$ of all genotypes, respectively, followed by the $\varepsilon 3 / \varepsilon 4(18.2 \%$ and 9.1\%, respectively), $\varepsilon 2 / \varepsilon 3$ ( $13.0 \%$ and $12.7 \%), \varepsilon 2 / \varepsilon 4$ (1.3\% and $2.3 \%), \varepsilon 4 / \varepsilon 4(0.6 \%$ and $0.6 \%)$ and $\varepsilon 2 / \varepsilon 2$ $(0.3 \%$ and $0.6 \%)$, in which $\varepsilon 3$ allele was the most common ( $82.1 \%$ and $85.6 \%)$, followed by $\varepsilon 4(10.4 \%$ and $6.3 \%)$ and $\varepsilon 2(7.5 \%$ and $8.1 \%)$. The distributions of ApoE genotypes

Table 2 The Distributions of Genotypes and Alleles of the ApoE Gene in the CAD Patients and Controls



Notes: ${ }^{\mathrm{a}} \mathrm{p}$ and $\mathrm{OR}(95 \% \mathrm{Cl})$ values were calculated by logistic regression adjusted for age, gender, and traditional cardiovascular risk factors. ${ }^{b} p$ values were calculated from two-sided chi-square tests or Fisher's exact tests.

Abbreviations: CAD, coronary artery disease; HWE, Hardy-Weinberg equilibrium. 
and alleles significantly differed between patients with CAD and the control participants $\left(\chi^{2}=11.93\right.$ and $P=0.04$, and $\chi^{2}=7.72$ and $P=0.02$, respectively).

Patients with $\mathrm{CAD}$ had a significantly lower $\varepsilon 3 / \varepsilon 3$ frequency (odds ratio $[\mathrm{OR}]=0.68,95 \%$ confidence interval $[\mathrm{CI}]=0.48-0.96, P=0.027)$ than did the control participants. Patients with CAD had significantly higher $\varepsilon 3 / \varepsilon 4(\mathrm{OR}=2.22,95 \% \mathrm{CI}=1.37-3.61, P=0.001)$ and $\varepsilon 4$ allele $(\mathrm{OR}=1.72,95 \% \mathrm{CI}=1.13-2.60, P=0.01)$ frequencies than did the control participants (Table 2).

Stratified analysis was further used to explore the effects of sex and age on the relationships. We analysed ApoE genotype frequencies in patients with $\mathrm{CAD}$ and control participants according to age (dichotomized into $\leq 60$ years and $>60$ years), which indicated that $\varepsilon 3 / \varepsilon 4$ frequency was significantly higher in patients with $\mathrm{CAD}$ ( $\leq 60$ years and $>60$ years old) than in the healthy control participants. $\varepsilon 4$ frequency was significantly higher in young patients with $\mathrm{CAD}$ (age $\leq 60$ years) than in the control participants, whereas no marked difference was indicated between elderly patients with CAD and the controls. Subsequent analysis of the data stratified by sex indicated that neither male nor female patients with CAD exhibited significant differences in genotype and allele frequencies compared with the control participants of both sexes (Table 3).

\section{Comparing the Clinical Data of Different ApoE Alleles Between the CAD Group and Control Participants}

Tables 4 and 5 list the associations between serum lipid profiles and allelic carrier statuses ( $\varepsilon 2, \varepsilon 3$ and $\varepsilon 4$ groups). $\varepsilon 2$ and $\varepsilon 4$ were considered to have opposite roles in lipid metabolism, and so participants with the $\varepsilon 2 / \varepsilon 4$ genotype were excluded. In the control participants, $\varepsilon 4$ carriers had significantly higher levels of TC, LDL-C, TG, ApoB and ApoB/ApoA-I, and lower levels of ApoE than did $\varepsilon 2$ carriers. However, ApoE polymorphisms did not significantly impact the levels of TC, HDL, LDL-C and TG in patients with $\mathrm{CAD}$. Moreover, $\varepsilon 4$ carriers had significantly higher levels of ApoB, ApoB/ApoA-I and lower levels of ApoE than did $\varepsilon 2$ carriers; $\varepsilon 2$ carriers also had lower, ApoB/ApoA-I and ApoE levels than did $\varepsilon 3$ and $\varepsilon 4$ carriers among patients with CAD.

\section{Logistic Regression Analysis of the Risk of CAD in the Northwest Population}

Univariate analysis indicated that the risk factors of smoking, drinking, diabetes mellitus, hypertension, low levels of HDL, ApoA-I and ApoE and high levels of TG and $\mathrm{Cr}$ were significantly associated with the presence of CAD $(P<0.05)$. We also performed a multivariate logistic regression analysis, which indicated that $\varepsilon 4$ allele (OR $=0.965,95 \% \mathrm{CI}=0.949-0.981 ; P=0.000)$, drinking, smoking, hypertension, TG and ApoE levels were significant and independent risk factors for CAD (Table 6). After stratifying by age ( $\leq 60$ years and $>60$ years) and sex, multivariate logistic regression analysis was performed. This indicated that $\varepsilon 4$ was a significant and independent risk factor for elderly patients with CAD (age $>60$ years: $\mathrm{OR}=3.30,95 \% \mathrm{CI}=1.10-9.86, P=0.033)$, but not for young (age $\leq 60$ years: $\mathrm{OR}=1.43,95 \% \mathrm{CI}=0.69-2.96$, $P=1.427)$, female $(\mathrm{OR}=1.15,95 \% \mathrm{CI}=0.36-3.65, P=$ $0.817)$ or male $(\mathrm{OR}=1.30,95 \% \mathrm{CI}=0.71-2.38, P=$ 0.403) patients with CAD (Table 7).

\section{Discussion}

CAD is a complex multifactorial disease modulated by multiple genetic and environmental factors, and contributes greatly to global morbidity and mortality. With changes to lifestyle and extensions to lifespan, the CAD prevalence is increasing sharply in China. ${ }^{10}$

ApoE is a $34 \mathrm{kDa}$ protein consisting of 299 amino acids responsible for lipid metabolism, and participates in TG transport and cholesterol metabolism. ${ }^{11}$ Previous studies have demonstrated how ApoE polymorphisms could affect ApoE gene transcription and cholesterol and TG levels, thus altering atherosclerosis progression, which is the main underlying pathology of CAD. ${ }^{12}$ Additional studies have also suggested ApoE may also increase the risk of CAD through the interaction with the antioxidative and immune system. ${ }^{13}$ Numerous previous studies have demonstrated that ApoE genotypes and allele frequencies vary significantly across different populations. ${ }^{14,15}$ The $\varepsilon 3$ allele is predominant in all populations, and $\varepsilon 2$ is thought to induce some effects protecting against CAD. ${ }^{12} \varepsilon 4$ has been identified across Europe at different frequencies, gradually decreasing from north to south. For example, the frequency of $\varepsilon 4$ ranges from more than $22 \%$ in Finland and Greenland to less than $7 \%$ in Greece and Italy. ${ }^{16,17}$ Epidemiological studies conducted in Asia (Iran, India, Singapore, Japan, China and Saudi Arabia) also found that $\varepsilon 3$ is the most prevalent subtype $(87 \%)$, while $\varepsilon 2$ and $\varepsilon 4$ have low prevalence rates in Asian countries. ${ }^{16}$ The order of frequencies of ApoE alleles in China is $\varepsilon 3$ $>\varepsilon 4>\varepsilon 2$, but there are also some other variations between north and south areas. ${ }^{14}$ 


\begin{tabular}{|c|c|c|c|}
\hline 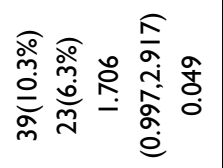 & 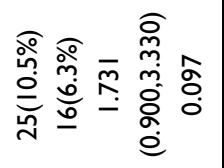 &  &  \\
\hline 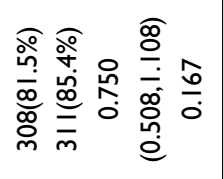 & 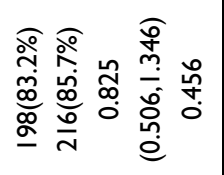 & 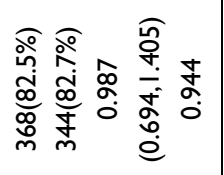 & 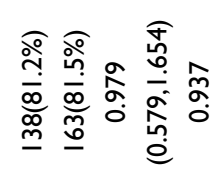 \\
\hline  & 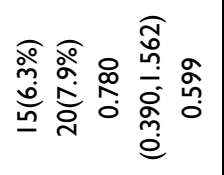 & 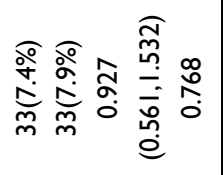 &  \\
\hline 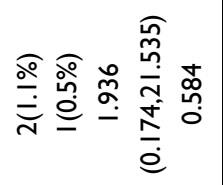 & 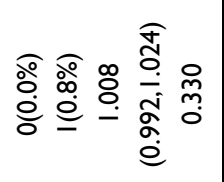 & 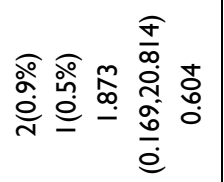 &  \\
\hline 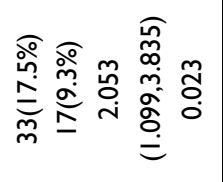 & 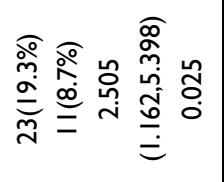 & 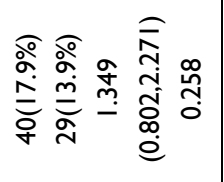 & 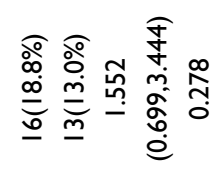 \\
\hline  &  & 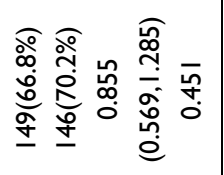 & 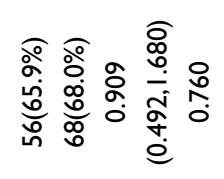 \\
\hline  & 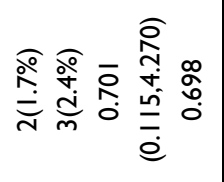 & 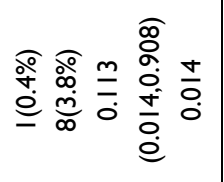 &  \\
\hline 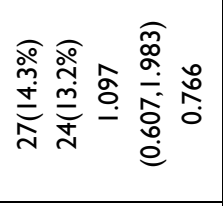 &  & 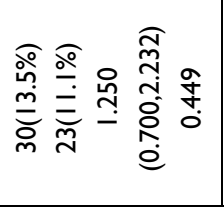 & 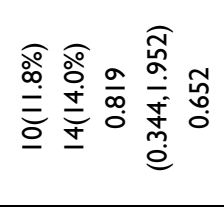 \\
\hline 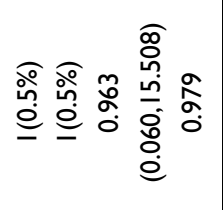 & 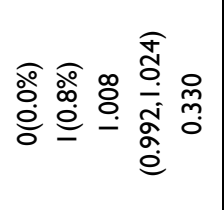 & 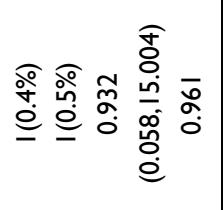 &  \\
\hline  & 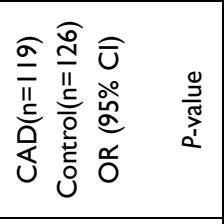 & 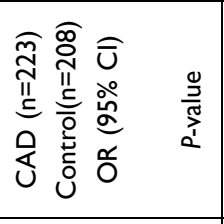 &  \\
\hline $\begin{array}{l}\text { O } \\
\text { vil } \\
\text { o. } \\
<\end{array}$ & $\begin{array}{l}\stackrel{0}{0} \\
\hat{\Lambda} \\
\stackrel{8}{<}\end{array}$ & $\frac{\tilde{y}}{\Sigma}$ &  \\
\hline
\end{tabular}


Table 4 Relationships Between Serum Lipid Profile and ApoE Allele in Control Participants

\begin{tabular}{|c|c|c|c|c|c|c|c|}
\hline Lipid Level (mmol/L) & $\varepsilon 2(\varepsilon 2 \varepsilon 2+\varepsilon 2 \varepsilon 3) n=4 \mid$ & $\varepsilon 3(\varepsilon 3) n=230$ & $\varepsilon 4(\varepsilon 3 \varepsilon 4+\varepsilon 4 \varepsilon 4) n=30$ & $P^{a}$ & $P^{b}$ & $P^{c}$ & $P^{d}$ \\
\hline TC & $3.6 I \pm 0.67$ & $3.80 \pm 0.86$ & $4.0 I \pm 0.82$ & $0.182^{\mathrm{a}}$ & 0.235 & 0.038 & 0.159 \\
\hline HDL & $1.04 \pm 0.21$ & $1.01 \pm 0.25$ & $1.03 \pm 0.25$ & $0.383^{\mathrm{a}}$ & 0.592 & 0.854 & 0.622 \\
\hline LDL-C & $1.82 \pm 0.58$ & $2.13 \pm 0.80$ & $2.27 \pm 1.01$ & $0.020^{\mathrm{a}}$ & 0.386 & 0.001 & 0.038 \\
\hline TG & $I .4 I \pm 0.59$ & $1.44 \pm 0.83$ & $1.83 \pm 0.82$ & $0.824^{\mathrm{a}}$ & 0.015 & 0.015 & 0.037 \\
\hline $\mathrm{Cr}$ & $61.86 \pm 15.54$ & $64.38 \pm 19.98$ & $66.94 \pm 27.53$ & $0.448^{\mathrm{a}}$ & 0.533 & 0.337 & 0.583 \\
\hline ApoA-I & $1.20 \pm 0.19$ & $1.15 \pm 0.19$ & $1.17 \pm 0.17$ & $0.121^{\mathrm{a}}$ & 0.510 & 0.558 & 0.265 \\
\hline ApoB & $0.67 \pm 0.22$ & $0.77 \pm 0.20$ & $0.92 \pm 0.22$ & $0.006^{\mathrm{a}}$ & 0.001 & 0.000 & 0.000 \\
\hline ApoB/ApoA-I & $0.58 \pm 0.24$ & $0.69 \pm 0.19$ & $0.79 \pm 0.19$ & $0.004^{\mathrm{a}}$ & 0.014 & 0.001 & 0.000 \\
\hline ApoE & $48.52 \pm 15.93$ & $36.06 \pm 12.87$ & $39.53 \pm 11.45$ & $0.000^{\mathrm{a}}$ & 0.163 & 0.012 & 0.000 \\
\hline
\end{tabular}

Notes: ${ }^{a} p$ values obtained when comparing $\varepsilon 2$ subjects with $\varepsilon 3$ subjects. ${ }^{b} p$ values obtained when comparing $\varepsilon 4$ subjects with $\varepsilon 3$ subjects. ${ }^{c} p$ values obtained when comparing $\varepsilon 2$ subjects with $\varepsilon 4$ subjects. ${ }^{d} p$ value shows the differences compared between groups $(\varepsilon 2, \varepsilon 3, \varepsilon 4)$.

Abbreviations: CAD, coronary artery disease; TC, total cholesterol; HDL, high-density lipoproteins; LDL-C, low-density lipoprotein cholesterol; TG, triglyceride; Cr, creatinine.

Table 5 Relationships Between Serum Lipid Profile and ApoE Allele in CAD Patients

\begin{tabular}{|c|c|c|c|c|c|c|c|}
\hline Lipid Level (mmol/L) & $\varepsilon 2(\varepsilon 2 \varepsilon 2+\varepsilon 2 \varepsilon 3) n=4 \mid$ & $\varepsilon 3(\varepsilon 3) n=230$ & $\varepsilon 4(\varepsilon 3 \varepsilon 4+\varepsilon 4 \varepsilon 4) n=30$ & $P^{a}$ & $P^{b}$ & $P^{c}$ & $P^{d}$ \\
\hline TC & $3.64 \pm 1.06$ & $3.77 \pm 1.09$ & $3.88 \pm 1.09$ & 0.491 & 0.463 & 0.255 & 0.531 \\
\hline HDL & $0.96 \pm 0.21$ & $0.96 \pm 0.19$ & $0.94 \pm 0.20$ & 0.838 & 0.650 & 0.630 & 0.865 \\
\hline LDL-C & $2.06 \pm 0.91$ & $2.21 \pm 1.01$ & $2.34 \pm 1.00$ & 0.398 & 0.363 & 0.148 & 0.378 \\
\hline TG & $1.82 \pm 0.98$ & $1.66 \pm 1.31$ & $1.69 \pm 0.72$ & 0.478 & 0.857 & 0.543 & 0.772 \\
\hline $\mathrm{Cr}$ & $68.01 \pm|7.7|$ & $66.87 \pm 17.66$ & $69.75 \pm 16.21$ & 0.707 & 0.265 & 0.614 & 0.532 \\
\hline ApoA-I & $1.15 \pm 0.21$ & $1.12 \pm 0.17$ & $1.09 \pm 0.18$ & 0.245 & 0.316 & 0.110 & 0.238 \\
\hline Apo B & $0.69 \pm 0.23$ & $0.76 \pm 0.24$ & $0.8 I \pm 0.23$ & 0.059 & 0.191 & 0.009 & 0.037 \\
\hline ApoB/ApoA-I & $0.6 I \pm 0.23$ & $0.70 \pm 0.25$ & $0.75 \pm 0.23$ & 0.036 & 0.136 & 0.002 & 0.015 \\
\hline ApoE & $50.33 \pm 21.48$ & $33.29 \pm 14.13$ & $30.26 \pm 7.62$ & 0.000 & 0.128 & 0.000 & 0.000 \\
\hline
\end{tabular}

Notes: ${ }^{a} p$ values obtained when comparing $\varepsilon 2$ subjects with $\varepsilon 3$ subjects. ${ }^{b} p$ values obtained when comparing $\varepsilon 4$ subjects with $\varepsilon 3$ subjects. ${ }^{c} p$ values obtained when comparing $\varepsilon 2$ subjects with $\varepsilon 4$ subjects. ${ }^{d} p$ value shows the differences compared between groups $(\varepsilon 2, \varepsilon 3, \varepsilon 4)$.

Abbreviations: CAD, coronary artery disease; TC, total cholesterol; HDL, high-density lipoproteins; LDL-C, low-density lipoprotein cholesterol; TG, triglyceride; Cr, creatinine.

Table 6 Logistic Regression Analysis of the Risk of CAD in Northwest of China Population

\begin{tabular}{|l|c|c|}
\hline Variables & P- value & OR (95\% Cl) \\
\hline Age & 0.064 & $1.014(0.999-1.028)$ \\
Drinking & 0.045 & $1.555(1.010-2.395)$ \\
Smoking & 0.018 & $1.609(1.084-2.388)$ \\
Hypertension & 0.000 & $1.988(1.386-2.853)$ \\
TG & 0.000 & $1.625(1.280-2.132)$ \\
ApoE & 0.000 & $0.965(0.949-0.981)$ \\
$\varepsilon 4$ & 0.032 & $1.826(1.055-3.163)$ \\
\hline
\end{tabular}

Abbreviations: CAD, coronary artery disease; TG, triglyceride.

The present study investigated the prevalence of polymorphisms and ApoE frequency among patients with CAD in northwest China. The ApoE genotypes frequencies in the CAD group were $0.3 \%, 13.0 \%, 1.3 \%, 66.6 \%$, $18.2 \%$ and $0.6 \%$ for $\varepsilon 2 / \varepsilon 2, \varepsilon 2 / \varepsilon 3, \varepsilon 2 / \varepsilon 4, \varepsilon 3 / \varepsilon 3, \varepsilon 3 / \varepsilon 4$ and $\varepsilon 4 / \varepsilon 4$, respectively; the corresponding frequencies in the
Table 7 Multiple Logistic Regression Analysis for CAD Patients and Control Subjects

\begin{tabular}{|l|c|c|}
\hline \multicolumn{3}{|c|}{$\varepsilon 4$} \\
\hline & OR (95\% Cl) & P-value \\
\hline Age $\leq 60$ & $1.427(0.688,2.959)$ & 1.427 \\
Age $>60$ & $3.295(1.101,9.862)$ & 0.033 \\
Males & $1.295(0.706,2.376)$ & 0.403 \\
Females & $1.147(0.360,3.650)$ & 0.817 \\
\hline
\end{tabular}

Abbreviation: CAD, coronary artery disease.

control participants were $0.6 \%, 12.7 \%, 2.3 \%, 74.7 \%, 9.1 \%$ and $0.6 \%$. In patients with CAD and control participants, 83 had the highest frequency $(82.1 \%$ and $85.6 \%$, respectively), while those for $\varepsilon 2$ and $\varepsilon 4$ were $7.5 \%$ and $8.1 \%$, $10.4 \%$ and $6.3 \%$, respectively, which were consistent with previous local and foreign research results. ${ }^{2,14}$ Our study indicated that $\varepsilon 4$ was significantly more common in 
patients with CAD than in the control group. We also found that $\varepsilon 4$ increased the CAD risk 1.9-fold overall, and 3.3-fold among elderly patients with CAD but not in young patients with CAD of both sexes. These results were consistent with those of previous studies. ${ }^{18}$ While the association between ApoE polymorphisms and CAD risk in different populations have been assessed in numerous studies, the results remain inconclusive. ${ }^{2,18}$

ApoE is a plasma glycoprotein associated with lipid transport and cholesterol homeostasis. The precise cellular mechanisms which are responsible for the differences between $\varepsilon 2, \varepsilon 3$ and $\varepsilon 4$ are not fully understood, but $\varepsilon 4$ has a somewhat higher affinity for the LDL receptor than $\varepsilon 3$, while $\varepsilon 2$ bind less well to the receptor-only $2 \%$ of normal activity, which results in different plasma lipoprotein profiles. ${ }^{19} \varepsilon 4$ is associated with elevated levels of TC, LDL-C, TG and ApoB, lower plasma levels of ApoE, and an increased CAD incidence. ${ }^{20,21} \mathrm{~A}$ large meta-analysis demonstrated that $\varepsilon 2$ carriers have a $20 \%$ lower CAD risk compared with $\varepsilon 3$ or $\varepsilon 4$ carriers. ${ }^{22}$ The present study found that the patients in the CAD group had higher TG levels and lower HDL levels. Furthermore, logistic regression analysis indicated that TG level is a strong independent risk factor for $\mathrm{CAD}(\mathrm{OR}=1.652,95 \% \mathrm{CI}=1.280-2.132, P<0.001)$. High HDL levels have been well documented to exert a protective effect on CAD, and low plasma HDL elevated plasma TG levels are associated with an increased CAD risk. ${ }^{23,24}$ Our results indicated that $\varepsilon 2$ carriers had lower TC, LDL-C and TG levels than did $\varepsilon 3$ or $\varepsilon 4$ carriers among the control participants. However, no significant differences were found between $\varepsilon 2, \varepsilon 3$ or $\varepsilon 4$ carriers and plasma lipoprotein levels among patients with CAD. This is consistent with some previous studies $^{25,26}$ but conflicts with others. ${ }^{27}$ The associations between ApoE genetic polymorphisms with lipid profiles have been widely studied among patients with $C A D$, although they remain controversial. ${ }^{14}$ The results of varied exposures due to racial, genetic and environmental factors are likely to influence the effects of ApoE polymorphism on blood lipid profiles. Moreover, most of the present patients with $\mathrm{CAD}$ were already receiving lipid-lowering therapy at the time of inclusion, which might explain the lack of a significant correlation between serum lipid profiles and ApoE alleles in patients with CAD.

ApoA-I is a major structural and functional component of $\mathrm{HDL}$, and is considered to play an essential role in reducing CAD risk. ${ }^{28} \mathrm{ApoB}$ is the main apolipoprotein of chylomicrons and LDL-C, and may contribute to atherogenesis. ${ }^{29}$ The ApoB/ ApoA-I ratio was found to be associated with CAD risk. ${ }^{30,31}$ Recent prospective studies indicated that low ApoA-I levels, high ApoB levels and the ApoB/A-I ratio may be even better predictors of $\mathrm{CAD}$ risk than traditional lipid parameters. ${ }^{32,33}$ However, there are also some conflicting data in the literature. ${ }^{34}$ In the present study, the CAD group had significantly lower ApoA-I levels than the control group, while ApoB levels and the ApoB/ApoA-I ratios did not differ significantly between the two groups. However, no significant difference was observed in ApoA-I levels among $\varepsilon 2, \varepsilon 3$ and $\varepsilon 4$ carriers, and $\mathrm{ApoB} / \mathrm{ApoA}-\mathrm{I}$ ratios and $\mathrm{ApoB}$ levels were significantly lower in $\varepsilon 2$ carriers than in $\varepsilon 3$ or $\varepsilon 4$ carriers in both groups. These results were consistent with those reported previously for other populations. ${ }^{35} \mathrm{We}$ also observed that $\varepsilon 4$ carriers had a significantly lower ApoE in both groups, which was consistent with previous findings of the ApoE level being highest in $\varepsilon 2$ carriers and lowest in $\varepsilon 4$ carriers. ${ }^{19,36}$

\section{Limitations of This Study}

First, As this is a large retrospective study, so it is difficult to exclude the influence of diet, activities and drugs on blood lipid level, which may lead to certain bias. Second, We cannot exclude that undetected significant differences between ApoE allele and lipid profile may because of the small sample size and lack statistical power.

\section{Conclusions}

In conclusion, our study suggested that $\varepsilon 4$ allele is associated with increased risk of CAD in Northwest of China population. Stratified analysis confirms that this elevated risk in individuals aged $\leq 60$ years old. After stratified by age and gender, multivariate logistic regression model analysis showed that $\varepsilon 4$ was a significant and independent risk factors in elderly CAD patients (age $>60$ ). In addition, $\varepsilon 4$ allele is associated with elevated TC, LDL-C, TG, ApoB and ApoB/ ApoA, while $\varepsilon 2$ allele is related to increased ApoE level. Therefore, ApoE genotyping may be clinically useful to help identify individuals at high risk of CAD and suggesting strategies for preventive measures and curative therapies.

\section{Acknowledgments}

We gratefully acknowledge all sample donors who participated in this study.

\section{Author Contributions}

All authors made a significant contribution to the work reported, whether that is in the conception, study design, execution, acquisition of data, analysis and interpretation, or in all these areas; took part in drafting, revising or critically reviewing the article; gave final approval of the 
version to be published; have agreed on the journal to which the article has been submitted; and agree to be accountable for all aspects of the work.

\section{Funding}

This work was supported by the Key R \& D Plan Projects in Shaanxi Province (grant number 2021-SF-130 and No. 2021SF-226), the Foundation of the First Affiliated Hospital of Xi'an Jiaotong University (grant number 2020ZYTS-08 and No. 2019QN-14) and Xi'an Science and technology planning project (No. 20YXYJ0001 (5)).

\section{Disclosure}

The authors declare that they have no competing interests.

\section{References}

1. El Shamieh S, Costanian C, Kassir R, Visvkis-Siest S, BissarTadmouri N. APOE genotypes in Lebanon: distribution and association with hypercholesterolemia and Alzheimer's disease. Per Med. 2019;16(1):15-23. doi:10.2217/pme-2018-0067

2. Larifla L, Armand C, Bangou J, et al. Association of APOE gene polymorphism with lipid profile and coronary artery disease in afro-Caribbeans. PLoS One. 2017;12(7):e0181620. doi:10.1371/journal.pone. 0181620

3. Shao Y, Zhao T, Zhang W, et al. Presence of the apolipoprotein E-epsilon 4 allele is associated with an increased risk of sepsis progression. Sci Rep. 2020;10(1):15735. doi:10.1038/s41598-02072616-0

4. Chiang S-M, Ueng K-C, Yang Y-S. Gender differences in variables associated with dipeptidyl peptidase 4 genetic polymorphisms in coronary artery disease. Adv Clin Exp Med. 2020;29 (10):1181-1186. doi:10.17219/acem/126291

5. Dankner R, Ben Avraham S, Harats D, Chetrit A. ApoE genotype, lipid profile, exercise, and the associations with cardiovascular morbidity and 18-year mortality. $J$ Gerontol a Biol. 2020;75 (10):1887-1893. doi:10.1093/gerona/glz232

6. Limonova AS, Ershova AI, Meshkov AN, et al. Case report: hypertriglyceridemia and premature atherosclerosis in a patient with apolipoprotein E gene epsilon 2 epsilon 1 genotype. Front Cardiovasc Med. 2021;7585779. doi:10.3389/fcvm.2020.585779

7. Bos MM, de Vries L, Rensen PCN, et al. Apolipoprotein E genotype, lifestyle and coronary artery disease: gene-environment interaction analyses in the UK biobank population. Atherosclerosis. 2021;328:33-37. doi:10.1016/j.atherosclerosis.2021.05.014

8. Luo J-Q, Ren H, Banh HL, et al. The associations between apolipoprotein E gene epsilon2/epsilon3/epsilon4 polymorphisms and the risk of coronary artery disease in patients with type 2 diabetes mellitus. Front Physiol. 2017;81031. doi:10.3389/fphys.2017.01031

9. Zhang L, He S, Li Z, et al. Apolipoprotein E polymorphisms contribute to statin response in Chinese ASCVD patients with dyslipidemia. Lipids Health Dis. 2019;18129. doi:10.1186/s12944019-1069-5

10. Hou J, Deng Q, Guo X, Deng X, Zhong W, Zhong Z. Association between apolipoprotein $\mathrm{E}$ gene polymorphism and the risk of coronary artery disease in Hakka postmenopausal women in southern China. Lipids Health Dis. 2020;19(1):139. doi:10.1186/s12944-02001323-6
11. Papandreou C, More M, Bellamine A. Trimethylamine N-oxide in relation to cardiometabolic health-cause or effect? Nutrients. 2020;12 (5):1330. doi:10.3390/nu12051330

12. Pereira LC, Nascimento JCR, Rego JMC, et al. Apolipoprotein E, periodontal disease and the risk for atherosclerosis: a review. Arch Oral Biol. 2019;98:204-212. doi:10.1016/j.archoralbio.2018.11.009

13. Gomez-Coronado D, Entrala A, Alvarez JJ, et al. Influence of apolipoprotein E polymorphism on plasma vitamin A and vitamin E levels. Eur J Clin Invest. 2002;32(4):251-258. doi:10.1046/j.13652362.2002.00983.x

14. Wang C, Yan W, Wang H, Zhu J, Chen H. APOE polymorphism is associated with blood lipid and serum uric acid metabolism in hypertension or coronary heart disease in a Chinese population. Pharmacogenomics. 2019;20(14):1021-1031. doi:10.2217/pgs-20190048

15. Berkinbayev S, Rysuly M, Mussayev A, et al. Apolipoprotein gene polymorphisms (APOB, APOC111, APOE) in the development of coronary heart disease in ethnic groups of Kazakhstan. J Genet Syndr Gene Ther. 2014;5(2):216. doi:10.4172/2157-7412.100021610.4172/ 2157-7412.1000216

16. Gerdes LU. The common polymorphism of apolipoprotein E: geographical aspects and new pathophysiological relations. Clin Chem Lab Med. 2003;41(5):628-631. doi:10.1515/cclm.2003.094

17. Adler G, Adler MA, Urbanska A, et al. Bosnian study of APOE distribution (BOSAD): a comparison with other European populations. Ann Hum Biol. 2017;44(6):568-573. doi:10.1080/ 03014460.2017.1346708

18. Zhao QR, Lei YY, Li J, Jiang N, Shi JP. Association between apolipoprotein E polymorphisms and premature coronary artery disease: a meta-analysis. Clin Chem Lab Med. 2017;55(2):284-298. doi:10.1515/cclm-2016-0145

19. Czaplinska M, Cwiklinska A, Sakowicz-Burkiewicz M, et al. Apolipoprotein E gene polymorphism and renal function are associated with apolipoprotein $\mathrm{E}$ concentration in patients with chronic kidney disease. Lipids Health Dis. 2019;1860. doi:10.1186/s12944019-1003-X

20. Zhao X-N, Sun Q, Cao Y-Q, Ran X, Cao Y. Association between apolipoprotein gene polymorphisms and hyperlipidemia: a meta-analysis. Bmc Genomic Data. 2021;22(1):14. doi:10.1186/ s12863-021-00968-1

21. El-Lebedy D, Raslan HM, Mohammed AM. Apolipoprotein E gene polymorphism and risk of type 2 diabetes and cardiovascular disease (vol 15, pg 12, 2016). Cardiovasc Diabetol. 2016;1535. doi:10.1186/ s12933-016-0354-0

22. Ren C, Baccarelli A, Wilker E, et al. Lipid and endothelium-related genes, ambient particulate matter, and heart rate variability-The VA Normative Aging Study. J Epidemiol Community Health. 2010;64 (1):49-56. doi:10.1136/jech.2008.083295

23. Wu Y-E, Ma L, Zhang H, Chen X-R, Xu X-Y, Hu Z-P. Significant association between the endothelial lipase gene 584C/T polymorphism and coronary artery disease risk. Biosci Rep. 2020;40Bsr20200027. doi: $10.1042 / \mathrm{bsr} 20200027$

24. Feng X, Guo Q, Zhou S, et al. Could remnant-like particle cholesterol become a risk factor in diabetic menopausal women with coronary artery disease? A cross-sectional study of single academic center in China. Lipids Health Dis. 2020;19(1):44. doi:10.1186/s12944-020-01224-8

25. Akanji AO, Suresh CG, Fatania HR, Al-Radwan R, Zubaid M. Associations of apolipoprotein E polymorphism with low-density lipoprotein size and subfraction profiles in Arab patients with coronary heart disease. Metab Clin Exp. 2007;56(4):484-490. doi:10.1016/j.metabol.2006.11.006

26. Ferreira CN, Carvalho MG, Fernandes APSM, et al. Comparative study of apolipoprotein-E polymorphism and plasma lipid levels in dyslipidemic and asymptomatic subjects, and their implication in cardio/cerebro-vascular disorders. Neurochem Int. 2010;56 (1):177-182. doi:10.1016/j.neuint.2009.09.016 
27. Abd El-Aziz TA, Mohamed RH. LDLR, ApoB and ApoE genes polymorphisms and classical risk factors in premature coronary artery disease. Gene. 2016;590(2):263-269. doi:10.1016/j.gene.2016.05.032

28. Banaganapalli B, Mansour H, Mohammed A, et al. Exploring celiac disease candidate pathways by global gene expression profiling and gene network cluster analysis. Sci Rep. 2020;10(1):16290. doi:10.1038/s41598-020-73288-6

29. Miura Y, Suzuki Y, Kanamaru H, et al. Higher non-fasting serum triglyceride preceding the carotid stenosis progression. Neurol Med Chir. 2021;61(7):422-432. doi:10.2176/nmc.oa.2020-0430

30. Galal H, Samir A, Shehata M. Assessment of apolipoprotein B/apolipoprotein A-I ratio in non-ST segment elevation acute coronary syndrome patients. Egypt Heart J. 2020;72(1):27. doi:10.1186/s43044-020-00057-1

31. Zheng S, Han T, Xu H, et al. Associations of apolipoprotein B/ apolipoprotein A-I ratio with pre-diabetes and diabetes risks: a cross-sectional study in Chinese adults. BMJ Open. 2017;7(1): e014038. doi:10.1136/bmjopen-2016-014038

32. Mazidi M, Katsiki N, Mikhailidis DP, et al. Apolipoprotein B/apolipoprotein A-I ratio is a better predictor of cancer mortality compared with C-reactive protein: results from two multi-ethnic US populations. J Clin Med. 2020;9(1):170. doi:10.3390/jcm9010170
33. Tian M, Li R, Shan Z, Wang DW, Jiang J, Cui G. Comparison of apolipoprotein B/A1 ratio, Framingham risk score and TC/HDL-c for predicting clinical outcomes in patients undergoing percutaneous coronary intervention. Lipids Health Dis. 2019;18(1):202. doi:10.1186/s12944-019-1144-y

34. Ingelsson E, Schaefer EJ, Contois JH, et al. Clinical utility of different lipid measures for prediction of coronary heart disease in men and women. J Am Med Assoc. 2007;298(7):776-785. doi:10.1001/ jama.298.7.776

35. Han S, Xu Y, Gao M, et al. Serum apolipoprotein E concentration and polymorphism influence serum lipid levels in Chinese Shandong Han population. Medicine. 2016;95(50):e5639. doi:10.1097/md.0000 000000005639

36. Wildsmith KR, Basak JM, Patterson BW, et al. In vivo human apolipoprotein e isoform fractional turnover rates in the CNS. PLoS One. 2012;7(6):e38013. doi:10.1371/journal.pone.0038013

\section{Publish your work in this journal}

Pharmacogenomics and Personalized Medicine is an international, peer-reviewed, open access journal characterizing the influence of genotype on pharmacology leading to the development of personalized treatment programs and individualized drug selection for improved safety, efficacy and sustainability. This journal is indexed on the American Chemical Society's Chemical Abstracts Service (CAS). The manuscript management system is completely online and includes a very quick and fair peer-review system, which is all easy to use. Visit http://www.dovepress.com/testimonials.php to read real quotes from published authors. 